\title{
Influence of Impurity Spin Dynamics on Quantum Transport in Epitaxial Graphene
}

\author{
Samuel Lara-Avila and Sergey Kubatkin \\ Department of Microtechnology and Nanoscience, Chalmers University of Technology, Göteborg S-412 96, Sweden \\ Oleksiy Kashuba \\ Institute of Theoretical Physics, Technische Universität Dresden, Dresden 01062, Germany \\ Joshua A. Folk and Silvia Lüscher \\ Quantum Matter Institute, University of British Columbia, Vancouver, British Columbia V6T 1Z4, Canada \\ and Department of Physics and Astronomy, University of British Columbia, Vancouver, British Columbia V6T 1Z1, Canada \\ Rositza Yakimova \\ Department of Physics, Chemistry and Biology (IFM), Linköping University, Linköping S-581 83, Sweden \\ T. J. B. M. Janssen \\ National Physical Laboratory, Teddington TW11 OLW, United Kingdom \\ Alexander Tzalenchuk* \\ National Physical Laboratory, Teddington TW11 OLW, United Kingdom \\ and Royal Holloway, University of London, Egham TW20 OEX, United Kingdom \\ Vladimir Fal'ko \\ Physics Department, Lancaster University, Lancaster LA1 4YB, United Kingdom
}

(Received 17 February 2015; published 3 September 2015)

\begin{abstract}
Experimental evidence from both spin-valve and quantum transport measurements points towards unexpectedly fast spin relaxation in graphene. We report magnetotransport studies of epitaxial graphene on $\mathrm{SiC}$ in a vector magnetic field showing that spin relaxation, detected using weak-localization analysis, is suppressed by an in-plane magnetic field $B_{\|}$, and thereby proving that it is caused at least in part by spinful scatterers. A nonmonotonic dependence of the effective decoherence rate on $B_{\|}$reveals the intricate role of the scatterers' spin dynamics in forming the interference correction to the conductivity, an effect that has gone unnoticed in earlier weak localization studies.
\end{abstract}

PACS numbers: 72.80.Vp, 73.20.Fz, 73.43.Qt, 75.76.+j

Loss of quantum information carried in the phase and spin of electrons propagating in a disordered conductor is associated with decoherence that suppresses interference corrections to the conductivity [1,2]. The fundamental relation between spin relaxation and the low-temperature magnetoresistance (MR) has been investigated theoretically and experimentally in numerous disordered metallic and semiconductor structures.

Recent studies of spin-valve [3-6] and quantum transport [7-11] effects in graphene-based devices have returned unexpectedly fast spin relaxation in graphene, despite this one-carbon-atom-thin material being billed as an ideal medium for spintronics applications [12-14]. Spin relaxation can be induced by either spin-orbit coupling or spinflip scattering due to magnetic impurities. In quantum transport, spin-orbit coupling inverts the conventional negative MR around zero perpendicular field $B_{\perp}$ (weak localization, WL) to positive MR known as weak antilocalization, but this effect has never been observed in pristine exfoliated or epitaxial graphenes [7-10,15,16]. By contrast, spin-flip scattering on magnetic impurities washes out quantum interference effects [17-21], including weak (anti-)localization. Spin-flip scattering in disordered metals has been shown [22-24] to raise the electron decoherence rate $\tau_{\varphi}^{-1}(T)$ above the value expected from inelastic scattering on thermal charge fluctuations [25],

$$
\tau_{T}^{-1}=\left(k_{B} T / \hbar\right)\left(\rho e^{2} / h\right) \ln \left(h / 2 e^{2} \rho\right) \equiv A T,
$$

where $\rho$ is resistivity. Residual decoherence in the limit $T \rightarrow 0$ is then determined only by the electron spin relaxation time $\tau_{s}$. Spin-flip decoherence was previously observed in exfoliated graphene [10]. Applying a large inplane magnetic field, $B_{\|} \gtrsim B_{T} \equiv k_{B} T / g_{i} \mu_{B}$, polarizes impurity spins with $g$ factor $g_{i}$, suppressing spin-flip scattering and prolonging phase coherence [10]. For magnetic fields too small to polarize impurity spins $B_{\|}<$ $B_{T}$ one might expect a minimal effect on decoherence.

In this Letter we show both experimentally and theoretically that such small in-plane fields do in fact have a 
discernable effect on the phase coherence, but the effect is opposite to that observed for larger $B_{\|}$. The measurement is performed on epitaxial graphene grown on silicon carbide ( $\mathrm{SiC} / \mathrm{G})$, using curvature of the $B_{\perp}$ MR peak to quantify the electron decoherence rate. Applying an in-plane magnetic field first broadens the MR peak slightly (enhances decoherence), before the sharpening effect due to impurity polarization sets in. This magnetic field dependence shows that the observed decoherence is caused by spin-flip scattering rather than other dynamical sources of decoherence, such as external noise due to external twolevel systems [26]. The nonmonotonic dependence of the decoherence rate on $B_{\|}$has not, to our knowledge, been discussed in previous work. It is a generic feature of quantum transport in disordered conductors that can be attributed to the precession of impurity spins at a frequency different from the spin precession of mobile electrons.

When electron $(e)$ and impurity $(i) g$ factors differ, the difference between their spin precession frequencies $\omega_{e \mid i}=$ $g_{e \mid i} \mu_{B} B_{\|} / \hbar$ leads to a random variation of the spin-dependent scattering conditions for electron waves retracing the same closed diffusive trajectory in clockwise and anticlockwise directions, whose interference forms the quantum correction to conductivity. The nonmonotonicity is characterized by a magnetic field scale, $B_{*}=\hbar \tau_{s}^{-1} /\left|g_{e}-g_{i}\right| \mu_{B}$, above which the decohering effect of the in-plane field (separating precession frequencies for impurities and conduction electrons) is overcome by polarization of impurity spins. The $g$ factor of magnetic scatterers can thus be determined by fitting the temperature and $B_{\|}$dependence of the MR curvature to theory developed below.

Magnetotransport measurements were performed on a large area $[160 \mu \mathrm{m} \times 35 \mu \mathrm{m}$, Fig. 1(a) $] n$-doped $\mathrm{SiC} / \mathrm{G}$ Hall bar, encapsulated in a polymer to improve temporal stability and doping homogeneity and exposed to deep-UV light to reduce carrier concentration $(n=10 \pm$ $1 \times 10^{11} \mathrm{~cm}^{-2}$ ) [27]. The device was measured at UBC in a dilution refrigerator equipped with a two-axis magnet, allowing independent control of $B_{\perp}$ and $B_{\|}$[10]. Average MR measurements are not obscured by mesoscopic conductance fluctuations due to the large sample size.

Figure 1(b) shows the characteristic negative MR of weak localization, measured for $B_{\|}=0$. As expected, MR is sharpest at the lowest temperatures where thermal charge fluctuations are minimized [Eq. (1)]. Raising the inplane field to $B_{\|}=1 \mathrm{~T}$ yields a significantly sharper (and higher) MR peak due to the partial polarization of impurity spins at this field [Fig. 1(c)]. Even in the raw data of Fig. 1(c), however, the nonmonotonicity that is the primary subject of this Letter can be clearly seen: the MR peak for $B_{\|}=0.3 \mathrm{~T}$ is broader than the trace at $B_{\|}=0$, despite the small but nonzero impurity polarization at this low inplane field.

To make further progress, the decoherence time $\tau_{\varphi}$ is quantified using the expression for the curvature $\kappa$ of magneto-conductivity around $B_{\perp}=0$, (a)

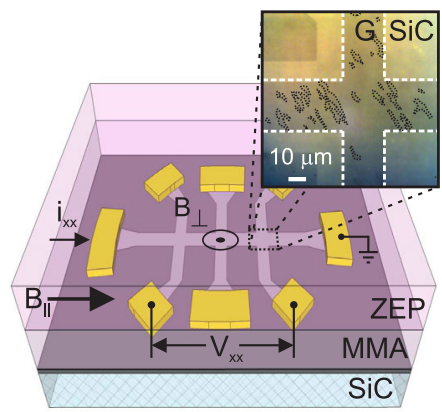

(b)

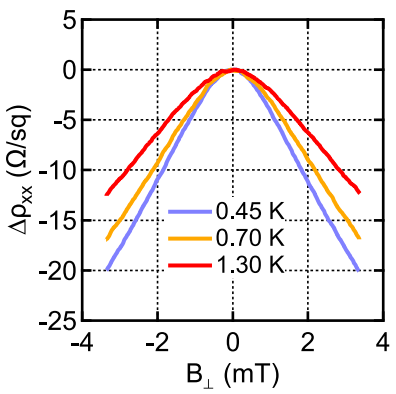

(c)

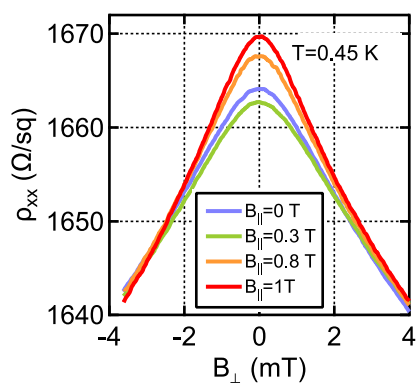

FIG. 1 (color online). (a) Layout of the sample and an optical micrograph [28] showing predominantly monolayer graphene with bilayer inclusions. (b) Magnetoresistance sharpens with decreasing temperature $\left(B_{\|}=0\right)$. (c) The sharpness of the magnetoresistance curve is nonmonotonic in $B_{\|}(T=450 \mathrm{mK})$.

$$
\left.\kappa \equiv \frac{\partial^{2} \sigma}{\partial B_{\perp}^{2}}\right|_{B_{\perp}=0}=\frac{16 \pi}{3} \frac{e^{2}}{h}\left(\frac{D \tau_{\varphi}}{h / e}\right)^{2}
$$

which comes from the basic functional form of WL $[1,2,29]$. Curvature is extracted from a parabolic fit to the average of 10 measurements of $\rho\left(B_{\perp}\right)$ covering the range of $\left|B_{\perp}\right| \leq 0.5 \mathrm{mT}$. The resulting temperature dependence of $\tau_{\varphi}^{-1}(T)$ at $B_{\|}=0$ clearly shows the linear scaling expected from Eq. (1) [Fig. 2(a)].

The slope $A$ of the temperature dependence in Eq. (1), estimated using $\rho_{x x}=1500 \pm 35 \Omega$, which we determine from the measured resistance and the sample aspect ratio, yields $A_{e}=16.4 \mathrm{~K}^{-1} \mathrm{~ns}^{-1}$ as compared to $A \approx$ $31 \mathrm{~K}^{-1} \mathrm{~ns}^{-1}$ fitted to experimentally determined $\tau_{\varphi}^{-1}(T)$ [Fig. 2(a)]. This difference can be reconciled in two ways. (i) Large area epitaxial graphene monolayers usually contain bilayer inclusions, which we also identified in the device used here, [Fig. 1(a)], and which have much higher conductivity than that of the monolayer material [30-33]. This reduces the effective length of the Hall bar, and changes the aspect ratio that should be used to calculate $\rho_{x x}$, and thereby $A$. (ii) The parameter $A$ may be treated simply as an empirical factor [15]. For the purposes of this Letter we simply rescale the effective sample area to force the slope of $\tau_{\varphi}^{-1}(T)$ to match that predicted by Eq. (1), giving $\rho_{x x} \sim 2800 \Omega$, mean free path $\sim 26 \mathrm{~nm}$, diffusion constant $D=131 \pm 10 \mathrm{~cm}^{2} / \mathrm{s}$, and the slope 
(a)

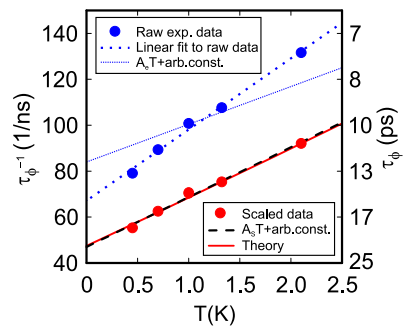

(b)

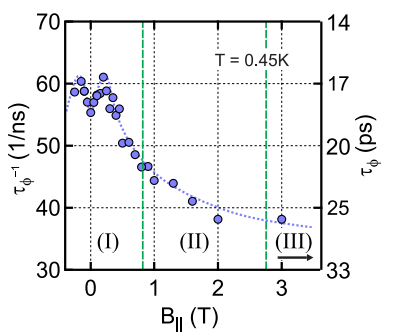

FIG. 2 (color online). (a) Dephasing rate as a function of temperature at $B_{\|}=0$ extracted from the raw (blue circles) and rescaled (red circles) magnetoresistance. The blue dotted line is the best linear fit to the raw data. The slopes of the dashed lines are given by Eq. (1), and the offset constants are chosen arbitrarily for ease of comparison with the data. The solid red line is the theory developed in this work. (b) Dephasing rate extracted from the rescaled magnetoresistance plotted as a function of $B_{\|}$at $T=450 \mathrm{mK}$. Roman numerals denote different scattering regimes. The dotted line is a guide to the eye.

$A_{s} \approx 22 \mathrm{~K}^{-1} \mathrm{~ns}^{-1}$. The black dashed line in Fig. 2(a) shows the plot of Eq. (1) for the rescaled geometry, which closely matches the rescaled data and extrapolates to a finite value corresponding to the decoherence time $\sim 20$ ps as $T \rightarrow 0$. Data presented in the remainder of this Letter are based on the rescaled area, but we point out that the alternative approach (ii) to treat the parameter $A$ does not alter the qualitative result of the following analysis of the quantum transport data.

Figure 2(b) shows $\tau_{\varphi}^{-1}$, extracted from the curvature of rescaled magnetoconductance, for different values of $B_{\|}$ $(T=450 \mathrm{mK})$. The observed dependence $\tau_{\varphi}^{-1}\left(B_{\|}\right)$features three characteristic regimes, labelled (I), (II), and (III). For intermediate fields (II), the polarization of spin-scattering impurities at $g_{i} \mu_{B} B_{\|} \gtrsim k_{B} T$ suppresses spin-flip scattering and decreases $\tau_{\varphi}^{-1}$. This prolongation of phase coherence by the in-plane field is a smoking gun for spin-flip scattering in the system. The suppression of $\tau_{\varphi}^{-1}$ is by a factor of approximately 2 before it begins to saturate at high field, indicating the entry into region III. The high-field saturation may be explained by flexural deformation of graphene out of the plane, resulting in randomly varying flux from the in-plane magnetic field [34], by $g \sim 0$ magnetic moments that are not polarized by magnetic field, or by spin-orbit interaction. This regime will be the subject of future work.

In the analysis that follows, we focus on the lowest-field regime (I), where the decoherence rate extracted using Eq. (2) shows a nonmonotonic behavior. Early studies of WL in dilute magnetic alloys demonstrated a "spin-memory" effect $[2,35]$ in the way the spinful scatterers reduce the size of the WL correction to conductivity. In summary, the exchange interaction between a spinful impurity and the electron spin $\mathbf{s}$, via a perturbation $J \mathbf{S} \cdot \mathbf{S} \delta\left(\mathbf{r}-\mathbf{r}_{i}\right)$, affects the electrons scattering in two ways: (a) by scattering an electron without flipping its spin and (b) by scattering with a spin flip. Although the spin flip process (b) always leads to the decoherence of electrons, process (a) leads only to additional scattering phases for a fixed spin configuration of impurities (relative to the electron spin). These phases would in general be the same for the two reversed sequences of visited scatterers; hence, they would not suppress the interference correction to conductivity. However, if the spin configuration of impurities is randomly changing in time, e.g., by Korringa relaxation, electron waves traveling along closed paths in opposite directions and therefore arriving at the same scatterer at different times would experience randomly different conditions for a spin-conserving scattering ("ergodic regime"), hence acquiring random relative phase shifts that suppress their interference contribution [35].

In contrast to Korringa relaxation, spin precession of impurity spins does not necessarily randomize spindependent scattering conditions for diffusing electron waves. If electrons and scatterers have equal $g$ factors $g_{i}=g_{e} \approx 2$, their precessions in an external magnetic field are in phase and, because scattering conditions depend only on the relative orientation of the two spins, the spinmemory regime would also persist in a finite $B_{\|}$. For scatterers with $g_{i} \neq g_{e}$, on the other hand, spin-dependent elastic scattering amplitudes for electrons following clockwise and anticlockwise trajectories can be partly destroyed by even a small $B_{\|}$. As sketched in Fig. 3(a), relative orientations of the spins of a scatterer and of the electron waves arriving along clockwise and anticlockwise trajectories responsible for the interference correction to conductivity would typically deviate by an angle $\left(g_{e}-g_{i}\right) \mu_{B} B_{\|} \tau_{\varphi} / \hbar$ and become randomized when $\left(g_{e}-g_{i}\right) \mu_{B} B_{\|}>h \tau_{\varphi}^{-1}$. This effect should be strongest in systems where the difference between the electron and scatterer $g$ factors is largest (in particular, in the systems where these two have opposite signs). This leaves a range of magnetic field where precessional decoherence can fully (a)

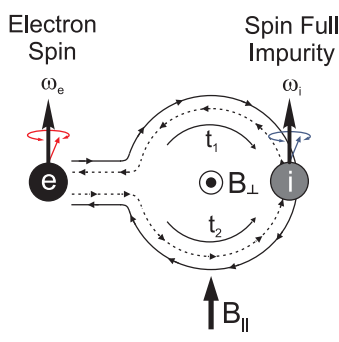

(b)

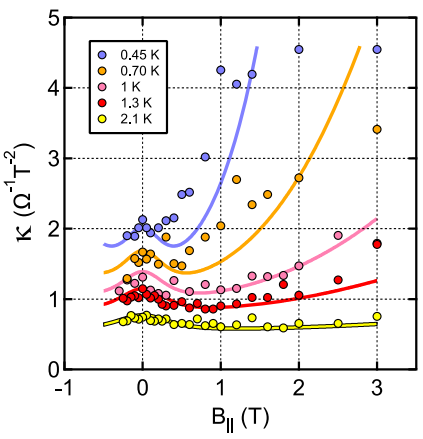

FIG. 3 (color online). (a) Illustration of the influence of precession of the impurity spin on the weak localization effect. (b) Comparison between the experimental values of the curvature of magnetoconductance at $T=0.45-2.1 \mathrm{~K}$ with the theoretical values calculated using the parameters obtained using a fit at $T=2.1 \mathrm{~K}$. 
develop (the ergodic regime) before the scatterers' spins become closely aligned with the external field $\left(B_{\|}>B_{T}\right)$, thus diminishing the role of precessional dynamics.

In WL theory, the difference between these spin-memory and ergodic regimes is accounted for by numerical factors that appear in the relations between dephasing rates $\tau_{S, M}^{-1}$ of two-electron correlators, Cooperons, and the electron scattering rate from the spinful impurity, $\tau_{s}^{-1}=$ $2 \pi \gamma n_{i} J^{2} S(S+1)$. Here, $\gamma=\left(1 / 2 \hbar^{2} v\right) \sqrt{\left(n_{e} / \pi\right)}$ stands for the density of states of carriers and $v$ is Dirac velocity of electrons in graphene. The Cooperons are classified according to their total spin $S=0,1$ and its projection $M$ onto the direction of external magnetic field. These four Cooperons $C_{S, M}$ are combined in the expression [2] for the WL correction to conductivity [36],

$$
\delta \sigma=\frac{e^{2}}{2 \pi h}\left[C_{0,0}-C_{1,0}-C_{1,1}-C_{1,-1}\right] .
$$

In the spin-memory regime, $\tau_{0,0}^{-1}=2 \tau_{s}^{-1}+\tau_{T}^{-1}$ and $\tau_{1, M}^{-1}=\frac{2}{3} \tau_{s}^{-1}+\tau_{T}^{-1}$, whereas in the ergodic regime [35], all Cooperons decay with the same rate, $\tau_{S, M}^{-1}=\tau_{s}^{-1}+\tau_{T}^{-1}$. Taking into account a possible difference between spin precession of the electrons and scatterers (which we do by changing the spin coordinates into the frame rotating with the frequency $\omega_{e}$ around the direction of external magnetic field $\mathbf{B}_{\|}$), we find using diagrammatic perturbation theory [37] that the two Cooperons $C_{1, \pm 1}$ with $S=1$ and $M= \pm 1$ are decoupled from all others and decay at the rate,

$$
\tau_{1, \pm 1}^{-1}=\frac{1}{\tau_{s}}\left[1-\frac{\left\langle S_{z}^{2}\right\rangle \pm\left\langle S_{z}\right\rangle\left(1-2 n_{F}\left(\varepsilon_{\mp}\right)\right)}{S(S+1)}\right]+\tau_{T}^{-1},
$$

where $\varepsilon_{\mp}=\varepsilon \pm g_{i} \mu_{B} B / 2$ takes into account that spin-flip scattering requires energy transfer to the scatterer. At the same time, spin precession mixes the two Cooperons, $C_{0,0}$ and $C_{1,0}$. This mixing generates combined modes, which relax with the decoherence rates

$$
\begin{aligned}
\tau_{0, \pm}^{-1}= & {\left[1+\frac{\left\langle S_{z}^{2}\right\rangle-\left\langle S_{z}\right\rangle\left[n_{F}\left(\varepsilon_{+}\right)-n_{F}\left(\varepsilon_{-}\right)\right]}{S(S+1)}\right] \tau_{s}^{-1} } \\
& \pm \sqrt{\tau_{1,+1}^{-1} \tau_{1,-1}^{-1}-\left(g_{e}-g_{i}\right)^{2} \mu_{B}^{2} B_{\|}^{2} / \hbar^{2}}+\tau_{T}^{-1} .
\end{aligned}
$$

Note that, at $B=0,\left\langle S_{z}\right\rangle=0$, and $\left\langle S_{z}^{2}\right\rangle=\frac{1}{3} S(S+1)$, so that $\tau_{1, \pm 1}^{-1}=\tau_{0,-}^{-1}=\frac{2}{3} \tau_{s}^{-1}+\tau_{T}^{-1}$ and $\tau_{0,+}^{-1}=2 \tau_{s}^{-1}+\tau_{T}^{-1}$, corresponding to the relaxation rates of triplet and singlet Coperons in the spin-memory regime. At $B_{\|}>B_{T}$, $\tau_{1, \pm 1}^{-1}=\tau_{T}^{-1}$, reflecting the restoration of phase coherence of diffusion of spin-polarized electrons.

Together, the four Cooperon modes [36] yield

$$
\begin{aligned}
\delta \sigma & =\frac{e^{2}}{2 \pi h} \int d \varepsilon \sum_{\alpha= \pm} n_{F}^{\prime}\left(\varepsilon_{\alpha}\right)\left[\ln \frac{\tau_{1, \alpha}}{\tau_{i v}}+A_{\alpha} \ln \frac{\tau_{0,-}}{\tau_{0,+}}\right], \\
A_{ \pm} & =\left(\tau_{1, \pm 1}^{-1}-\tau_{T}^{-1}\right) /\left(\tau_{0,+}^{-1}-\tau_{0,-}^{-1}\right),
\end{aligned}
$$

and magnetoconductance (measured as a function of $B_{\perp}$ for fixed $\left.B_{\|}\right)$,

$$
\begin{aligned}
\sigma\left(B_{\perp}, B_{\|}\right)= & \sigma\left(0, B_{\|}\right)-\frac{e^{2}}{2 \pi h} \int d \varepsilon \sum_{\alpha= \pm} n_{F}^{\prime}\left(\varepsilon_{\alpha}\right) \\
& \times\left\{F\left(\frac{B_{\perp}}{B_{1, \alpha}}\right)+\left[F\left(\frac{B_{\perp}}{B_{0,-}}\right)-F\left(\frac{B_{\perp}}{B_{0,+}}\right)\right] A_{\alpha}\right\} . \\
F(z)= & \ln z+\psi\left(\frac{1}{2}+\frac{1}{z}\right) ; \quad B_{\beta, \alpha}=\frac{\hbar / 4 e}{D \tau_{\beta, \alpha}},
\end{aligned}
$$

where $n_{F}^{\prime}(\varepsilon) \equiv \partial n_{F}(\varepsilon) / \partial \varepsilon$ is a derivative of the Fermi distribution function, $\psi$ is the digamma function, $\tau^{-1}$ is the momentum relaxation rate that determines the classical Drude conductivity and the diffusion coefficient, $D=\frac{1}{2} v^{2} \tau$ (note that for monolayer graphene studied in this work, $D \approx 131 \mathrm{~cm}^{2} / \mathrm{s}$ ), and $\tau_{i v}$ is the intervalley scattering time $[36,39]$.

A nonmonotonic dependence of the decoherence rate on the in-plane magnetic field follows from the calculated magnetoconductance curvature,

$$
\begin{aligned}
\kappa= & \frac{2 e^{2}}{3 \pi h} \int d \varepsilon \sum_{\alpha= \pm}\left(-n_{F}^{\prime}\left(\varepsilon_{\alpha}\right)\right) \\
& \times\left\{\left(\frac{D \tau_{1, \alpha}}{\hbar / e}\right)^{2}+\left(\tau_{1, \pm 1}^{-1}-\tau_{T}^{-1}\right)\left(\tau_{0,+}+\tau_{0,-}\right) \frac{D \tau_{0,+}}{\hbar / e} \frac{D \tau_{0,-}}{\hbar / e}\right\} .
\end{aligned}
$$

Here, we used the expansion $F(z) \approx z^{2} / 24+O\left[z^{3}\right]$.

Equation (4) gives a semiquantitative description of the observed magnetoconductance curvature that captures its qualitative features over a wide range of temperatures 0.45 $2.1 \mathrm{~K}$ and in-plane magnetic fields $(0-3 \mathrm{~T})$ [Fig. 3(b)]. Here, the following protocol was used for a single fit that yielded all five curves shown in Fig. 3(b): (1) The temperature dependence of magnetoconductance curvature $\kappa$ at $B_{\|}=0$ is used to extract $\tau_{s}^{-1} \approx 77 \mathrm{~ns}^{-1}$ and the parameter $A_{t} \approx 24 \mathrm{~ns}^{-1} \mathrm{~K}^{-1}$ from the fit to our theory to be compared with $A_{s} \approx 22 \mathrm{~ns}^{-1} \mathrm{~K}^{-1}$ from Eq. (1). The red line in Fig. 2(a) is the theoretical temperature dependence, which nearly coincides with the straight dashed line given by Eq. (1) and perfectly matches the experimental points. (2) The dependence of $\kappa$ on $B_{\|}$at high temperature, $T=$ 2.1 K [bottom data set in Fig. 3(b)], is used to determine the average $g$ factor of the scatterers, which returned the value $g_{i}=-0.84 \pm 0.25$.

The parameters determined above are sufficient to calculate the $B_{\|}$dependence of the magnetoconductance curvature for lower temperatures, down to $T=450 \mathrm{mK}$, which are then compared with the experimentally measured values [Fig. 3(b)]. The theoretically calculated curvature (lines) captures the observed nonmonotonic behavior and a shift of the low-field anomaly towards smaller $B_{\|}$at lower temperatures [40].

To conclude, we have demonstrated that the excess decoherence rate, observed earlier at low temperatures in epitaxial graphene sublimated on $\mathrm{SiC}[7,8]$, is caused by the 
spin-flip scattering of electrons from spinful impurities. These spinful scatterers have an average $g$ factor $g_{i}=-0.84 \pm 0.25$, very different from the free-electron $g$ factor in graphene, $g_{e} \approx 2$, which enabled us to observe the influence of precession of the impurities'spins on the WL effect. The large (and negative) $g$ factor for the impurity spin implies a strong atomic spin-orbit coupling in the magnetic moment formation. This may indicate that these spins are in the surface states underneath the graphene layer, possibly originating in $\mathrm{Si}$ substitutions of carbon atoms in the interfacial layer [41]. The presence of such spinful scatterers on the $\mathrm{SiC}$ surface, directly accessible for graphene electrons, explains the short spin coherence length observed in spinvalve experiments on a similar material [6].

We thank I. Aleiner, L. Glazman, C. Marcus, B. van Wees, and A. MacDonald for useful discussions. This work has been supported by NSERC, CFI, Quantum Matter Institute (QMI), the EC Graphene Flagship (CNECT-ICT604391), European Metrology Research Program (EMRP) project GraphOhm, ERC Synergy Grant Hetero2D, Deutsche Forschungsgemeinschaft through Research Training Group GRK 1621, Linnaeus Centre for Quantum Engineering, Swedish Research Council, Knut and Alice Wallenberg Foundation, Swedish Foundation for Strategic Research (SSF), and Chalmers Area of Advance NANO.

*alexander.tzalenchuk@npl.co.uk

[1] B. L. Altshuler, D. Khmel'nitzkii, A. I. Larkin, and P. A. Lee, Phys. Rev. B 22, 5142 (1980).

[2] S. Hikami, A. I. Larkin, and Y. Nagaoka, Prog. Theor. Phys. 63, 707 (1980).

[3] T. Y. Yang et al., Phys. Rev. Lett. 107, 047206 (2011).

[4] T. Maassen, J. J. van den Berg, N. IJbema, F. Fromm, T. Seyller, R. Yakimova, and B. J. van Wees, Nano Lett. 12, 1498 (2012).

[5] B. Dlubak et al., Nat. Phys. 8, 557 (2012).

[6] T. Maassen, J. J. van den Berg, E. H. Huisman, H. Dijkstra, F. Fromm, T. Seyller, and B. J. van Wees, Phys. Rev. Lett. 110, 067209 (2013).

[7] S. Lara-Avila, A. Tzalenchuk, S. Kubatkin, R. Yakimova, T. J. B. M. Janssen, K. Cedergren, T. Bergsten, and V. Fal'ko, Phys. Rev. Lett. 107, 166602 (2011).

[8] J. Jobst, D. Waldmann, I. V. Gornyi, A. D. Mirlin, and H. B. Weber, Phys. Rev. Lett. 108, 106601 (2012).

[9] A. A. Kozikov, D. W. Horsell, E. McCann, and V. I. Fal'ko, Phys. Rev. B 86, 045436 (2012).

[10] M. B. Lundeberg, R. Yang, J. Renard, and J. A. Folk, Phys. Rev. Lett. 110, 156601 (2013).

[11] D. Smirnov, J. C. Rode, and R. J. Haug, Appl. Phys. Lett. 105, 082112 (2014).

[12] A. Avsar et al., Nano Lett. 11, 2363 (2011).

[13] D. Pesin and A. H. MacDonald, Nat. Mater. 11, 409 (2012).

[14] W. Han, R. K. Kawakami, M. Gmitra, and J. Fabian, Nat. Nanotechnol. 9, 794 (2014).
[15] F. V. Tikhonenko, D. W. Horsell, R. V. Gorbachev, and A. K. Savchenko, Phys. Rev. Lett. 100, 056802 (2008).

[16] F. V. Tikhonenko, A. A. Kozikov, A. K. Savchenko, and R. V. Gorbachev, Phys. Rev. Lett. 103, 226801 (2009).

[17] P. A. Lee, A. D. Stone, and H. Fukuyama, Phys. Rev. B 35, 1039 (1987).

[18] A. D. Stone, Phys. Rev. B 39, 10736 (1989).

[19] V. Chandrasekhar, P. Santhanam, and D. E. Prober, Phys. Rev. B 42, 6823 (1990).

[20] A. K. Geim, S. V. Dubonos, and I. Y. Antonova, JETP Lett. 52, 247 (1990).

[21] P. G. N. de Vegvar, L. P. Lévy, and T. A. Fulton, Phys. Rev. Lett. 66, 2380 (1991).

[22] A. B. Gougam, F. Pierre, H. Pothier, D. Esteve, and N. O. Birge, J. Low Temp. Phys. 118, 447 (2000).

[23] F. Pierre and N. O. Birge, Phys. Rev. Lett. 89, 206804 (2002).

[24] F. Pierre, A. B. Gougam, A. Anthore, H. Pothier, D. Esteve, and N. O. Birge, Phys. Rev. B 68, 085413 (2003).

[25] B. L. Altshuler and A. G. Aronov, in Electron-Electron Interaction in Disordered Systems, edited by A. L. Efros and M. Pollak (North-Holland, Amsterdam, 1985).

[26] J. J. Lin and J. P. Bird, J. Phys. Condens. Matter 14, R501 (2002).

[27] S. Lara-Avila, K. Moth-Poulsen, R. Yakimova, T. Bjørnholm, V. Fal'ko, A. Tzalenchuk, and S. Kubatkin, Adv. Mater. 23, 878 (2011).

[28] T. Yager et al., Nano Lett. 13, 4217 (2013).

[29] The conductivity used here is simply the inverse of measured resistivity, $\sigma=1 / \rho_{x x}$.

[30] F. Giannazzo, I. Deretzis, A. La Magna, F. Roccaforte, and R. Yakimova, Phys. Rev. B 86, 235422 (2012).

[31] V. Panchal, C. E. Giusca, A. Lartsev, R. Yakimova, and O. Kazakova, Front. Phys. 2, 3 (2014).

[32] T. Yager, A. Lartsev, R. Yakimova, S. Lara-Avila, and S. Kubatkin, Carbon 87,409 (2015).

[33] S. Kopylov, A. Tzalenchuk, S. Kubatkin, and V. I. Fal'ko, Appl. Phys. Lett. 97, 112109 (2010).

[34] M. B. Lundeberg and J. A. Folk, Phys. Rev. Lett. 105, 146804 (2010).

[35] V. I. Fal'ko, JETP Lett. 53, 340 (1991).

[36] In graphene one may also take into account valley degeneracy of electrons, and, hence, consider both valley-singlet and triplet Cooperons [39]. However, in the presence of intervalley scattering, which is the necessary prerequisite [39] for the observation of WL (rather than suppressed weak antilocalization), only valley-singlet Cooperons need to be taken into account.

[37] See Supplemental Material at http://link.aps.org/ supplemental/10.1103/PhysRevLett.115.106602 for the details of the theoretical model, which includes Ref. [37].

[38] E. M. Lifshitz and L. P. Pitaevskii, Physical Kinetics (Elsevier, New York, 2006).

[39] E. McCann, K. Kechedzhi, V. I. Fal'ko, H. Suzuura, T. Ando, and B. L. Altshuler, Phys. Rev. Lett. 97, 146805 (2006).

[40] The above analysis was performed for the in-plane field range I and II, where the out-of-plane deformations of graphene do not play an important role.

[41] J. Hass, J. E. Millán-Otoya, P. N. First, and E. H. Conrad, Phys. Rev. B 78, 205424 (2008). 